\title{
Simulationstraining in der Hämodialyse Ein Weg zu bester Praxis in der Ausbildung
}

Thomas Fernsebner, Traunstein, stellte auf der 44. EDTNA/ERCA-Konferenz 2015 in Dresden sein Poster „Simulationstraining in der Hämodialyse - ein Weg zu bester Praxis in der Ausbildung“ vor.

Die Simulation ist eine Schulungsmethode, die zunächst als Sicherheitstraining in der Verkehrsindustrie (Flug-, Raumund Schifffahrt, Bahn und Transportwesen) das Sicherheits-Management-System (SMS: Safety Management System) sehr erfolgreich verbessert hat. Ebenso ist dieser Bereich in der Akut-, Notfall- und interventionellen Medizin, wie z.B. Intensivmedizin, Notfalleinheiten, OP, Gynäkologie u.v.m., nicht mehr weg zu denken [1].

\section{Simulationen werden immer wichtiger}

In der Dialyse gab es bislang nur einfache Ansätze, die Simulation v.a. im Notfallbereich umzusetzen. Das Megacode-Training bezeichnet die Schulung und Übung eines strukturierten Vorgehens bei HerzLungen-Wiederbelebung im Sinne des Mega-Code-Algorithmus, nach den Richtlinien des ERC (European Resuscitation Council) [2]. Zweifelsfrei ist dieses Training ein wichtiger Baustein für mögliche Notfallsituationen und trägt zur Verbesserung in auftretenden Notfallkrisen für diese trainierten Ereignisse bei, wenn sichergestellt ist, dass das nephrologische Team vor Ort regelmäßig an diesen Trainings teilgenommen hat.

Aus der genannten Erfahrung heraus und aus den weiteren vielfältig möglichen Notfallereignissen in der Dialyse nahm

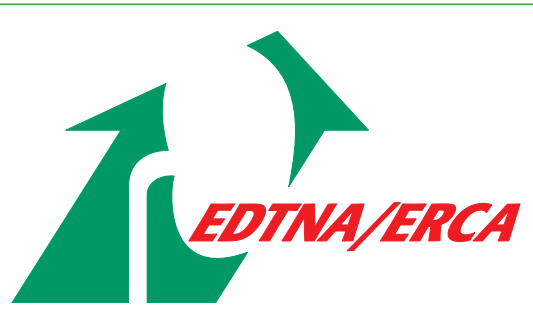

EDTNA/ERCA Brand-Ambassador Germany Thomas Fernsebner

Akademie nephrologischer Berufsgruppen e. K. Bürgerwaldstraße 1

83278 Traunstein

E-Mail: info@nephro-weiterbildung.de

Internet: www.edtnaerca.org die Akademie nephrologischer Berufsgruppen e.K., Traunstein, diese Herausforderung an und entwickelte ein eigenes Notfalltraining mit integriertem extrakorporalem Kreislauf.

Poster auf EDTNA|ERCA-Konferenz Auf der letztjährigen 44. Konferenz der EDTNA/ERCA in Dresden wurde erstmals diese Methode auf einer Konferenz gezeigt und stießen auf ein enormes Interesse [3]. Die Methode kombiniert bisherige Simulationsmöglichkeiten mit kardiovaskulären Ereignissen und mit dialyseassoziierten Notfällen wie:

- Volumenmangel,

- RR-Abfall,

- Schock,

- Herzstillstand,

- Hyperkaliämie,

- Blutverlust,

- Hämolyse,

- Flussprobleme u.v.m.

Weiter kann mit der Simulation das Basistraining für Dialyseanfänger, aber auch das Routinetraining eingeübt werden.

\section{Viele Vorteile}

Die Simulation übt Verhaltensweisen ohne Patienten, weshalb diese Übungssituation enorme ethische Vorteile bringt. Anfänger in der Nierenersatztherapie haben wenige Übungsmöglichkeiten im Trockentraining und sind häufig schnell am Patienten ersten Erfahrungen gegenübergestellt. Simulationen in der Dialyse könnten ein neuer Standard im Training und in der Schulung der Nierenersatztherapie sein und künftig weiterentwickelt werden.

Es wurden bereits mehrere Simulationen in der Akademie nephrologischer Berufsgruppen e.K. durchgeführt. Alle Personen aus den ersten Trainings wurden nach einem halben Jahr befragt. Das Resultat zeigte eine hohe Akzeptanz dieser Methode (Daten noch nicht veröffentlicht). Es ist denkbar, dass nephrologisches Personal aller Berufsgruppen sich künftig in unterschiedlichen Übungsszenarien der Simulation unterziehen sollte, um die Sicherheit des Patienten zu erhöhen.

Alois Gorke, Murnau

EDTNA/ERCA Supervisory Board Member 2016

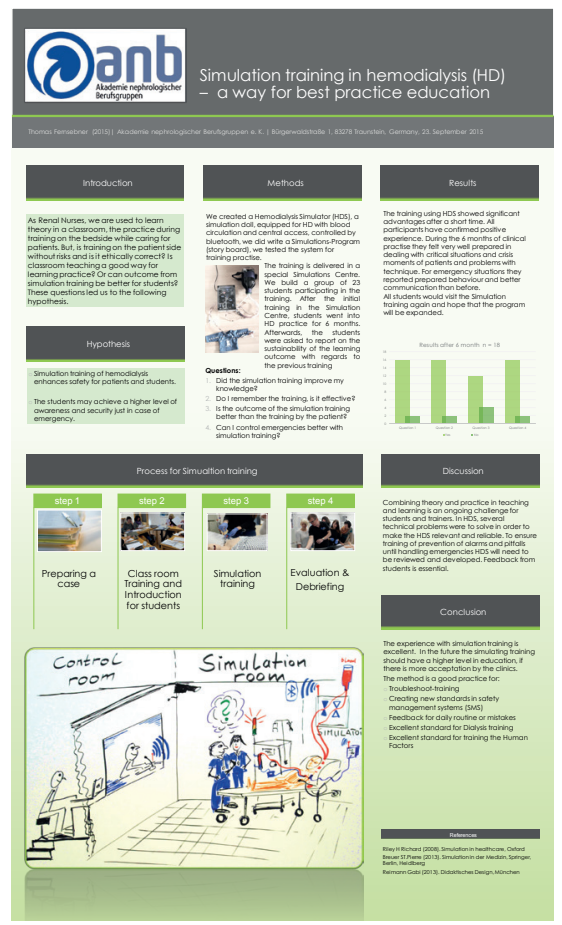

Abb. 1 Poster „Simulationstraining in der Hämodialyse - ein Weg zu bester Praxis in der Ausbildung“ von Thomas Fernsebner, Traunstein.

Literatur

1 St. Pierre M, Breuer G, Hrsg. Simulation in der Medizin: Grundlegende Konzepte - Klinische Anwendung. Berlin: Springer; 2013

2 Nolan JP, Deakin CD, Soar J et al.; European Resuscitation Council. European Resuscitation Council guidelines for resuscitation 2005. Section 4. Adult advanced life support. Resuscitation 2005; 67 (Suppl. 1): S39-S86

3 Fernsebner T. Simulation training in hemodialysis - a way for best practice education. Poster auf der 44. EDTNA/ERCA International Conference, 26.-29.09.2015, Dresden

\section{Einladung}

Das deutsche Netzwerk der EDTNA/ERCA verfolgt das Ziel, dass Innovationen, Erfahrungen und wissenschaftliche Erkenntnisse aus Deutschland in den Foren der EDTNA/ERCA eingereicht werden. Dies kann eine pflegewissenschaftliche Arbeit, ein Abstract als Vortrag oder Poster und/oder ein Beitrag in den Internetforen der EDTNA/ERCA sein. Beteiligen Sie sich an diesem Dialog und nehmen Sie Kontakt zur EDTNA/ERCA in Deutschland auf, wir helfen Ihnen gerne! 